[Vicino Oriente XVIII (2014), pp. 123-144]

\title{
NUOVE STELE DAL TOFET DI MOZIA*
}

\author{
Bruno D’Andrea - Università di Napoli «L’Orientale»
}

This paper analyses nine new votive stelae discovered during the archaeological investigations at the Tophet of Motya carried out in 2009 by the Regional Superintendence BB.CC.AA. of Trapani and the Rome «La Sapienza» Expedition to Motya.

Keywords: Phoenicians; Motya; tophet; Baal Hammon; stelae

\section{IL CONTESTO DI RITROVAMENTO}

Le indagini condotte nel 2009 nell'ambito dei lavori per il restauro e la musealizzazione del tofet dalla Soprintendenza BB.CC.AA. Regionale di Trapani e dalla Missione archeologica a Mozia dell'Università di Roma «La Sapienza» hanno consentito il rinvenimento di nove stele votive ${ }^{1}$. I reperti erano sempre in giacitura secondaria, come del resto la stragrande maggioranza delle stele del tofet ${ }^{2}$.

\section{MT.09.3011/S2}

Il monumento lapideo ${ }^{3}$, rinvenuto nel corso della pulizia preliminare del santuario (US.3011; fig. 1:1), è un piccolo cippo di arenaria spezzato alla sommità, in particolar modo sul lato destro, caratterizzato da varie scheggiature perimetrali e abrasioni superficiali, soprattutto alla base (fig. 2:1-3; alt. 17,8 cm; largh. 9,3 cm; spess. 3,8 - 8,8 $\mathrm{cm})$. La forma del cippo è quella di un trono con spalliera e braccioli aggettanti, il raccordo tra i due è ad angolo ottuso e, sulla faccia anteriore, è costituito da un gradino rilevato su cui è posto un betilo con base indistinta, leggermente rastremato, reso con un rilievo molto basso ottenuto attraverso un'ampia solcatura dei due lati. La tipologia dei cippi a trono con betilo è attestata nel repertorio lapideo moziese in una decina di casi ${ }^{4}$, alcuni dei quali

* Le stele esaminate in questo contributo sono state presentate dall'autore nel corso della IX Giornata Romana di Studi Moziesi Antonia Ciasca «From Sidon to Motya 2010» (Roma, 25 febbraio 2011). Ringrazio il Prof. Lorenzo Nigro per avermi concesso l'opportunità di studiare queste stele e per aver seguito la stesura dell'articolo.

1 Nigro 2012, 212-213, figg. 312-314. Alle indagini del 2009 è seguita una ripresa e un'estensione delle ricerche archeologiche al tofet da parte della Missione archeologica a Mozia dell’Università di Roma «La Sapienza». Queste ricerche, che continuano tuttora, hanno prodotto notevoli risultati per quanto riguarda l'architettura e la stratigrafia del santuario: Nigro 2013.

2 Solo in qualche occasione A. Ciasca ha segnalato, soprattutto per gli strati V e III (corrispondenti alle fasi 6 e 8 della stratigrafia del santuario proposta da L. Nigro sulla base dei recenti scavi: Nigro 2013, 39), l'esistenza di stele in situ deposte in connessione all'urna cineraria: Ciasca et al. 1964,, 52, tav. LIX; Brancoli et al. 1967 , 17, tavv. XI e XIII-XVI; Ciasca et al. 1970, 75, figg. 6-7, tavv. XL-XLI; Bevilaqua et al. 1972, 91; Ciasca et al. 1973, 85, tavv. LX-LXI. I casi in cui il rapporto diretto tra la deposizione della/e urna/e e quella della stele è riconoscibile sul terreno sono rari anche nei tofet del Nord Africa analizzati recentemente dall'autore: D’Andrea 2014, 296-297.

3 Per le stele votive, per le deposizioni e per il contenuto delle urne cinerarie, nel corso della missione del 2009 è stato elaborato e utilizzato un nuovo sistema di catalogazione e informatizzazione dei dati modellato sulle specifiche problematiche archeologiche poste dai santuari tofet.

4 Moscati - Uberti 1981, 112-115, nn. 188-210, tavv. XXIV-XXVIII. 
direttamente confrontabili con il reperto in esame (fig. 2:4) ${ }^{5}$. Quando il dato è disponibile, i cippi a trono di Mozia sono sistematicamente attribuiti allo strato $\mathrm{V}$, il primo strato del tofet in cui compaiono le stele votive corrispondente alla fase 8 della stratigrafia del santuario recentemente elaborata da L. Nigro (620-550 a.C. $)^{6}$. Questa tipologia formale corrisponde al tipo III,2 variante $a^{\beta}$ della classificazione proposta da $\mathrm{H}$. Bénichou-Safar per il tofet di Cartagine, dove viene datata tra il secondo quarto del VII sec. a.C. e una fase compresa tra la metà del VI e la fine del V sec. a.C. ${ }^{7}$; nel catalogo delle stele arcaiche del tofet di Cartagine di P. Bartoloni compaiono oltre cinquanta cippi-trono con betilo singolo ${ }^{8}$. La tipologia è attestata raramente nei repertori di Nora, Sulcis e, probabilmente, Monte Sirai ${ }^{9}$, mentre è assente a Sousse e Tharros ${ }^{10}$.

\section{MT.09.3011/S3}

Il reperto, recuperato nel corso della pulizia del santuario (fig. 1:2), appartiene alla stessa tipologia del primo, ma in questo caso il trono è privo di braccioli (fig. 3:1-3; alt. $28,5 \mathrm{~cm}$; largh. $16,1 \mathrm{~cm}$; spess. $6,3-16,9 \mathrm{~cm}$ ). Si tratta di un cippo di arenaria calcarea spezzato alla sommità e caratterizzato da scheggiature perimetrali e abrasioni superficiali; sulla faccia anteriore si riconoscono ampie tracce dell'originaria intonacatura. Il raccordo tra spalliera e base, quest'ultima aggettante di circa $3 \mathrm{~cm}$, è ad angolo ottuso, l'apparato illustrativo è costituito da un grosso betilo leggermente rastremato ottenuto attraverso una sottile incisione dei lati. Il reperto trova un confronto abbastanza stringente nel repertorio moziese (fig. 3:4), qualche altro confronto proviene dal tofet di Cartagine ${ }^{11}$. La modalità di resa del betilo, con sottile incisione dei quattro lati, è attestata su stele di tipo diverso a Cartagine, Mozia e Sulcis ${ }^{12}$

\section{MT.09.3011/S2}

Il cippo è stato rinvenuto appoggiato, probabilmente in epoca moderna, sui blocchi che costituivano il muro denominato G da A. Ciasca (fig. 1:9). Esso, di arenaria calcarea poco coerente, è pressoché integro ma presenta su tutte le facce, in particolar modo su quella laterale sinistra, alla base e alla sommità, rotture, abrasioni e scheggiature diffuse dovute anche alla scarsa qualità della roccia impiegata (fig. 4:1-3; alt. 19,1 cm; largh. 9,4 cm; spess. 9,2 - 17,4 cm). La forma è quella del cippo a trono con raccordo tra base e spalliera

5 Moscati - Uberti 1981, 113, n. 196, tav. XXVI (strato V); 114, n. 201, tav. XXVII.

6 Nigro 2013, 39 (cfr. Nigro 2004). A. Ciasca aveva proposto una datazione compresa tra il secondo e l'ultimo quarto del VI sec. a.C.: Ciasca 1992, 123-126 e 129.

7 Bénichou-Safar 2004a, 139-140 e 184-185, tav. LII, 3-5. Cfr. D’Andrea 2014, 19, fig. 1.2 (III, 2a2); 50-51; 55 .

8 Bartoloni 1976, 87-88, nn. 69-76, tavv. XIX-XXII; 88-92, nn. 79-125, tavv. XXIII-XXXVI. Cfr. 89, n. 90, tav. XXVI; 89, n. 92, tav. XXVI; 90, n. 104, tav. XXVII; 91, n. 108, tav. XXXI; 92, n. 123, tav. XXXV (= CMA, 161, Cb 513, tav. LXIV).

9 Moscati - Uberti 1970, 83, n. 2, fig. 1a, tav. I (Nora); Bondì 1972, 96, n. 1, fig. 1,1, tav. I (Monte Sirai); Bartoloni 1986, 38, nn. 66-69, tav. IX (Sulcis; cippi estremamente frammentari).

10 Per lo studio del repertorio lapideo di Sousse: D’Andrea 2014, 81-97. Per Tharros: Moscati - Uberti 1985.

11 Bartoloni 1976, 88-89, nn. 84-85, tav. XXIV.

12 Cfr. ad esempio Bartoloni 1976, 105, n. 226, tav. LXIV; 111, n. 281, tav. LXXVIII; Moscati - Uberti 1981, 139-147, nn. 352-409, tavv. LIII-LX; Bartoloni 1986, 43, n. 103, tav. XV; 47, n. 136, tav. XXI. 
ad angolo ottuso e la base presenta un aggetto di circa $5 \mathrm{~cm}$. L'apparato illustrativo è costituito da un betilo leggermente rastremato scolpito con un bassorilievo molto marcato, la cui base corrisponde a quella del trono. Soltanto un altro cippo moziese, attribuito allo strato $\mathrm{V}^{13}$, presenta un betilo rilevato alla stessa maniera, ma in questo caso la forma è un po' diversa, con raccordo tra spalliera e base ad angolo retto. Qualche altro confronto proviene da Cartagine ${ }^{14}$ e dall'unico cippo-trono del repertorio norense (fig. 4:4) ${ }^{15}$.

\section{MT.09.M3020/S4}

Il reperto è stato rinvenuto appoggiato su un grosso blocco facente parte di M. $3020^{16}$, ma la circostanza di ritrovamento non permette di affermare che fosse stato riutilizzato all'interno di questo muro in età antica (fig. 1:3). Si tratta di un cippo a trono di pietra calcarea caratterizzato da scheggiature, rotture e abrasioni superficiali più o meno ampie, del quale risultano mancanti la sommità e la parte anteriore della base (fig. 5:1-3; alt. 39,4 cm; largh. 20,9 cm; spess. 11,9 - 16,1 cm). Il reperto ha un profilo a forma di $\mathrm{L}$, la base aggetta attualmente di $2,5 \mathrm{~cm}$. La tipologia dei cippi a trono vuoti è ampiamente attestata nel repertorio moziese per tutta la sua durata (fig. 5:4-5) ${ }^{17}$, dallo strato $\mathrm{V}$ allo strato III (fasi 6-8 della nuova stratigrafia del santuario: $620-470$ a.C. $)^{18}$. Essa corrisponde alla variante $a^{\alpha}$ del tipo III,2 della classificazione proposta per Cartagine da H. Bénichou-Safar (fig. 5:7) ${ }^{19}$; è possibile che cippi di questa tipologia fossero in uso già nella parte finale della prima fase di vita del tofet cartaginese, cioè nel corso della prima metà del VII sec. a.C. Il tipo appare ben rappresentato nei repertori di Sulcis (fig. 5:6) e Tharros ${ }^{20}$, mentre è assente a Nora e Monte Sirai, dove la produzione lapidea comincia in una fase un po' più tarda ${ }^{21}$, nonché a Sousse, dove la produzione si caratterizza, soprattutto nelle prime fasi, per l'elaborazione di nuove tipologie formali ${ }^{22}$.

\section{MT.09.M3020/S3}

Come il cippo precedente, questo reperto è stato rinvenuto in M.3020 ma in questo caso esso appariva effettivamente reimpiegato nel muro ed è pertanto stato lasciato in situ (fig.

13 Moscati - Uberti 1981, 113, n. 194, tav. XXV.

14 Bartoloni 1976, 87, nn. 68-69, tavv. XIX-XX; 88, nn. 75-76, tavv. XXI-XXII; 88, n. 79, tav. XXIII.

15 Moscati - Uberti 1970, 83, n. 2, fig. 1a, tav. I. Sembra essere dello stesso tipo anche l'unico cippo a trono di Monte Sirai: Bondì 1972, 96, n. 1, fig. 1,1, tav. I.

16 Si tratta di un muretto ad andamento est/ovest, forse di terrazzamento, costituito da piccolo pietrame e stele votive riutilizzate, perlopiù in stato di conservazione frammentario. Oltre alle tre stele trattate nel presente articolo, nel muro era riutilizzata una quarta stele (MT.09.M3020/S2), lasciata in situ, il cui apparato illustrativo è caratterizzato da un disco solare e un betilo rastremato.

17 Moscati - Uberti 1981, 95-99, nn. 68-96, tavv. IX-XIV; 108-112, nn. 159-186, tavv. XX-XXIV.

18 Nigro 2013, 39 (cfr. Nigro 2004).

19 Bénichou-Safar 2004a, 139-140 e 184-185, tav. LII, 1-2. Cfr. D’Andrea 2014, 19, fig. 1.2 (III, 2a1); 46; 5051 .

20 Moscati - Uberti 1985, 97, nn. 18-19, tav. VI; 98-99, nn. 25-28, tavv. VIII-IX; Bartoloni 1986, 35-38, nn. 4265, tavv. VI-IX

21 A Nora non prima della fine del VI sec. a.C., quando sembra iniziare la vita del santuario; a Monte Sirai nel corso del IV sec. a.C.: D'Andrea - Giardino 2013.

22 Per le tipologie formali di Sousse: D’Andrea 2014, 81-83, tab. 3.1; 84-85; 87-89. 
$1: 4 ; 6: 1)$. Si tratta di una lastra di roccia calcarea giallastra, organogena, di cui risultano spezzati l'angolo inferiore sinistro e quello superiore destro (fig. 6:2-3; alt. 27,1 cm; largh. $16,7 \mathrm{~cm}$; spess. $6,5 \mathrm{~cm}$ ); la faccia anteriore reca resti di un'originaria intonacatura. L'apparato illustrativo è costituito da un grosso betilo a sezione semiellittica e sommità leggermente arrotondata ottenuto attraverso dei solchi abbastanza profondi scavati sui quattro lati, i quali al tempo stesso delimitano un'edicola di tipo semplice. La tipologia formale con edicola semplice a cornice è ampiamente attestata nel repertorio moziese (fig. $6: 4-5)$, con almeno tre varianti $(\mathrm{A}-\mathrm{C})^{23}$, mentre in quello cartaginese è molto più rara ${ }^{24}$; a Cartagine l'edicola, sovente egittizzante, è resa in maniera più dettagliata, spesso con inquadramenti plurimi, ed è provvista in genere di trabeazione, cella e basamento ${ }^{25}$. Il tipo trova pochi confronti anche nei repertori lapidei degli altri tofe ${ }^{26}$, meno che a Sulcis dove è ampiamente attestato come a Mozia ${ }^{27}$.

\section{MT.09.M3020/S1}

Il reperto è attribuibile alla stessa tipologia formale del cippo precedente, nello specifico alla variante C, e anch'esso era riutilizzato in M.3020 (fig. 1:5 Si tratta di una lastra di pietra calcarea grigio-verdastra, ricomposta da tre frammenti, integra ma caratterizzata da scheggiature profonde lungo la cornice esterna e da abrasioni superficiali diffuse (fig. 7:1-3; alt. 33,7 cm; largh. 30,7 cm; spess. 9,6 - 18,8 cm). La faccia anteriore è costituita da un'edicola a inquadramento semplice aggettante entro la quale è scolpito, con un bassorilievo non molto profondo, un betilo rastremato a sommità arrotondata.

\section{MT.09.M3035/S1}

La stele è stata rinvenuta appoggiata, probabilmente in età moderna, a uno dei blocchi del limite A (rinominato M.3035) della solida massicciata che nella prima fase del santuario costituiva una sorta di terrazza cultuale che poteva ospitare, secondo A. Ciasca, una piccola cappella egittizzante (fig. 1:6 ${ }^{28}$. Si tratta di una lastra di arenaria calcarea grossolana della

23 Nella variante A i limiti del betilo corrispondono grossomodo a quelli dell'edicola (fig. 8:4-5; cfr. Moscati Uberti 1981, 139-147, nn. 352-405, tavv. LIII-LX; 155-158, nn. 458-477, tavv. LXVII-LXX); nella variante B la base del betilo è indistinta e gli altri tre lati corrispondono a quelli dell'edicola (Moscati - Uberti 1981, 148153, nn. 415-443, tavv. LXI-LXV); nella variante C l'edicola semplice è distinta dal betilo (Moscati - Uberti 1981, 153, nn. 444-445, tav. LXV; 159, nn. 482-484, tav. LXXI; 162-163, nn. 502-505, tav. LXXIV; 164-165, nn. 514-518, tavv. LXXVI-LXXVII).

24 Per qualche esempio: Bartoloni 1976, 105, n. 222, tav. LXIII; 105, n. 226, tav. LXIV; 109, n. 264, tav LXXIV; n. 296, tav. LXXXII.

25 Cfr. Bénichou-Safar 2004a, 139 e 180-184, tav. L-LI (tipo III,1); D’Andrea 2014, 50, tav. I; 54-55. Questo tipo di edicola è ampiamente attestato anche nel repertorio moziese.

26 Ad esempio Moscati - Uberti 1970, 84, n. 3, tav. II; 88-89, n. 11, tav. VI; 90, n. 13, tav. VII.

27 Bartoloni 1986, 42-48, nn. 96-142, tavv. XIV-XXII (predomina la variante C).

28 Ciasca et al. 1964, 47-60; Ciasca 1992, 119; Nigro 2004, 41 (l'autore parla di un sacello quadrato). In realtà i dati archeologici non permettono di avanzare ricostruzioni certe sulla configurazione e sull'uso di questa terrazza, nella quale si avvicendarono sicuramente più interventi nel corso del tempo che ne cambiarono l'assetto originario; gli scavi in corso apporteranno informazioni fondamentali a questo proposito. La centralità e la sacralità dell'area all'interno del santuario è garantita dal fatto che essa è installata su uno sperone roccioso dominante rispetto alla piana circostante e che qui non furono rinvenute urne cinerarie. Le ricerche del 2009 hanno rivelato la presenza di un individuo adulto inumato proprio vicino all'importante 
quale si conserva poco meno della metà superiore (fig. 8:1-4; alt. $23 \mathrm{~cm}$; largh. 21,5 cm; spess. 5-6,5 cm). Il reperto, caratterizzato da scheggiature perimetrali e abrasioni superficiali diffuse, è di buona fattura: la pietra è tagliata e sbozzata con cura, la faccia anteriore è lisciata in maniera rifinita e reca tracce dell'originaria intonacatura. La forma è quella di una lastra rettangolare con taglio superiore piatto e depressione obliqua, sulla faccia anteriore si sviluppa un'edicola con pilastri raccordati in alto e sormontati da una trabeazione; quest'ultima è costituita in basso da una gola egizia posta tra listelli incisi, i quali si sviluppano anche nella parte centro-superiore. All'interno dell'edicola è scolpito con un rilievo basso e schiacciato un personaggio maschile stante, di profilo, rivolto a destra; di esso si conservano soltanto la parte superiore del busto e la testa, l'immagine risulta di difficile lettura a causa dell'abrasione del rilievo. Il personaggio, che indossa un copricapo conico, ha una lunga barba, il braccio destro piegato in alto con mano aperta e il braccio sinistro proteso in avanti.

7.1. L'iconografia del personaggio con copricapo conico, barba lunga e mano alzata: caratteri, confronti e proposte interpretative

La stele trova confronti puntuali nel repertorio moziese in una ventina di reperti (fig. $8: 5 ; 9: 1-4)$ pertinenti agli strati IV-III, dunque alle fasi 6-7 (550-470 a.C. $)^{29}$. Gli elementi che caratterizzano il personaggio moziese, attestato probabilmente anche nel tofet di Cartagine $^{30}$, sono la vista di profilo e la resa egittizzante con barba lunga, folta e in alcuni casi a punta, copricapo conico e braccio destro alzato con mano aperta in segno di saluto/giuramento ${ }^{31}$; il vestiario è costituito da una lunga veste che ricade sulla gamba posteriore, in due casi il personaggio sorregge un'asta terminante con qualcosa alla sommità e utilizzata come scettro ${ }^{32}$. Come proposto in passato da E. Acquaro e S. Moscati, il personaggio moziese può essere accostato a quello raffigurato su una stele di Sulcis datata al VI sec. a.C. (fig. 9:5) ${ }^{33}$ : in questo caso la figura sorregge un'asta terminante con un elemento lanceolato e interpretata come una lancia. Qualche altro esempio del tipo può essere costituito da una stele di VI sec. a.C. proveniente da Kouklia, purtroppo in pessimo

stipe votiva scavata da A. Ciasca tra i blocchi D ed E: R. Giglio - L. Nigro, Intervento di restauro al Tofet di Mozia: Ah. Ferjaoui (ed.), Actés du VII congrès international des études phéniciennes et puniques (Hammamet, 10-14 novembre 2009), Tunis (in stampa). Appena a est del muro B, a una quota molto più bassa perché il settore è posto alla base dello sperone roccioso, A. Ciasca rinvenne i resti di almeno cinque individui collocati l'uno sull'altro senza ordine apparente (Ciasca et al. 1964, 49). Tali resti si trovavano, probabilmente in giacitura secondaria, in un terreno compatto giallastro molto simile al terreno alluvionale e privo di ceramica; non si può escludere che tale terreno fosse dilavato dallo sperone sovrastante, considerando anche il fatto che il muro di contenimento B è sicuramente seriore ad A (Ciasca et al. 1964, 59-60). Per confronto, si può ricordare il ritrovamento di un individuo inumato nel tofet di Sousse associato a un'anfora di VI sec. a.C., dunque apparentemente contemporaneo alla vita del santuario (D’Andrea 2014, 76).

29 Per i confronti: Moscati - Uberti 1981, 242-246, nn. 920-940, tavv. CLXIV-CLXVIII; 248, n. 947, tav. CLXX; 248, n. 949, tav. CLXXI. Per la cronologia delle fasi 6-7: Nigro 2013, 39.

30 Bartoloni 1976, 146-147, n. 576, tav. CLVIII; 151, n. 605, tav. CLXV.

31 Per l'interpretazione del gesto cfr. Bénichou-Safar 2004b, 99-116; D'Andrea 2014, 62, nota 364.

32 Moscati - Uberti 1981, 248, n. 947, tav. CLXX (potrebbe trattarsi di uno scettro w3s); 248, n. 949, tav. CLXXI.

33 Moscati 1967, 63; Acquaro 1969, 69-72. Secondo E. Acquaro il lapicida non era riuscito a calcolare bene lo spazio per riprodurre il cartone che aveva a disposizione, da ciò l'assenza del copricapo conico. 
stato di conservazione (fig. 9:6) ${ }^{34}$, e da uno scarabeo di probabile provenienza cipriota, nel quale però il personaggio reca sulla testa una mitra (fig. $9: 7)^{35}$; un altro confronto, ipotetico in assenza delle braccia e considerando che in questo caso il torso è nudo, è costituito da un bronzetto di VII sec. a.C. proveniente da Sancti Petri (Cadice) ${ }^{36}$.

Potrebbe costituire una variante della resa stante quella, meglio testimoniata e canonizzata, presente probabilmente anche nel repertorio moziese, seppur in maniera estremamente stilizzata (fig. 10:1-2), nella quale un personaggio simile è assiso su un trono, in genere sorretto da sfingi alate. Questa iconografia fenicio-egittizzante è attestata in ambito orientale (fig. 10:4-7) ) $^{37}$ ma è nel mondo fenicio e punico d'Occidente che trova la sua massima diffusione. La cosiddetta stele di Baal del tofet di Sousse, databile al V sec. a.C., costituisce un'ottima esemplificazione del tipo (fig. 10:3): il personaggio è visto di profilo, la resa è egittizzante con barba lunga, copricapo conico, atteggiamento di saluto/giuramento, asta con elemento ellissoidale/lanceolato e trono sorretto da sfingi alate $^{38}$. Altri esempi consistono in alcuni scarabei: uno di diaspro verde-scuro montato su un anello d'argento (fig. 10:8) ${ }^{39}$ e un secondo di diaspro verde, entrambi provenienti da Ibiza (fig. 10:9) ${ }^{40}$; due scarabei, il primo di diaspro verde (fig. 10:10) ${ }^{41}$ e il secondo di agata (fig. 10:11) ${ }^{42}$, da Tharros; uno scarabeo di diaspro verde di probabile provenienza moziese (fig. $10: 12)^{43}$ e altri due di provenienza sconosciuta (fig. 10:13-14) ${ }^{44}$. La resa del personaggio è più stilizzata in un anello d'oro proveniente da Utica databile al IV-III sec. a.C. (fig. 10:15) ${ }^{45}$ e in uno scarabeo di diaspro verde da Sulcis (fig. 10:16) ${ }^{46}$. In altri casi lo stesso personaggio è assiso su un trono di tipo diverso, privo di sfingi alate (fig. 10:17$26)^{47}$, e può essere sprovvisto dell'asta (fig. 10:27-28) ${ }^{48}$. In relazione a quest'ultima, è

34 Wilson 1974, 142-146, tav. XXI, 6.

35 Acquaro 1969, 70-71, fig. 1. Secondo l'autore qui, come nella stele di Sulcis, il personaggio è interpretabile come Resheph.

36 Peters ed. 2004, 342, n. 19.

37 Ad esempio: uno scarabeo di diaspro rosso-bruno proveniente da Tiro (fig. 13:4 = Gubel 1987, 39-40, n. 6 , tav. IV); uno scarabeo di diaspro verde di provenienza sconosciuta, ma comunque dalla Fenicia (fig. 13:5 = Gubel 1987, 40, n. 7, tav. IV); una bulla, anch'essa dal territorio fenicio ma di provenienza sconosciuta (fig. 13:6 = Gubel 1987, 40-41, n. 9, tav. V); uno scarabeo di diaspro verde-scuro montato su un anello di bronzo dorato da Nicosia (fig. 13:7 = Boardman 2003, 63, n. 17/30, tav. 17; cfr. Gubel 1987, 42, n. 12, tav. VI).

38 Picard 1954, 298, Cb 1075, tav. CXXVI. Cfr. Bisi 1967, 94-96, fig. 56; D’Andrea 2014, 82, S. 2 , tav. VIII, 1.

39 Boardman 2003, 62, n. 17/1, tav. 16. Cfr. Gubel 1987, 43-44, n. 16, tav. VII.

40 Boardman 2003, 62, n. 17/2, tav. 16. Cfr. Gubel 1987, 44, n. 17, tav. VII.

41 Boardman 2003, 63, n. 17/8, tav. 16. Cfr. Gubel 1987, 44, n. 18, tav. VIII.

42 Boardman 2003, 65, n. 17/x13, tav. 53. Cfr. Gubel 1987, 44, n. 20, tav. VIII.

43 Gubel 1987, 47, n. 28, tav. IX.

44 Boardman 2003, 66, n. 17/x15, tav. 54 (corniola, Museo di Kassel); 66, n. 17/x17, tav. 54 (impressione conservata a Oxford).

45 Peters ed. 2004, 235, n. 13. Cfr. Gubel 1987, 42, n. 13, tav. VI; D’Andrea 2014, 148, fig. 6.2.

46 Gubel 1987, 44-45, n. 19, tav. VIII.

47 Ad esempio: un anello d'oro proveniente da Cartagine (fig. 13:17 = Culican 1970, 28-32, fig. I, b; cfr. Gubel 1987, 114, n. 51, tav. XX); uno scarabeo di diaspro verde ancora da Cartagine (fig. 13:18 = Boardman 2003 63, n. 17/24, tav. 16; cfr. Gubel 1987, 179, n. 130, tav. XXXVI); uno scarabeo di diaspro verde da Ibiza (fig. 13:19 = Boardman 2003, 63, n. 17/3, tav. 16; cfr. Gubel 1987, 142, n. 98, tav. XXXII); quattro scarabei da Tharros (fig. 13:20-23 = Peters ed. 2004, 183; Gubel 1987, 181, n. 141, tav. XXXVIII; 126, n. 58, tav. XXII; 
ipotizzabile che essa, sia quando termina superiormente con un elemento lanceolato o ellissoidale sia quando tale elemento è reso in maniera più particolareggiata, funga da scettro e costituisca un riferimento al mondo vegetale, una pianta su alto stelo tipo papiro, palma, bocciolo di loto o spiga di grano ${ }^{49}$; questa tipologia di scettri è ampiamente attestata già in ambito orientale, negli scarabei come, ad esempio, negli avori fenici di Nimrud ${ }^{50}$.

Il personaggio rappresentato nella variante in trono sembra essere una divinità, mentre resta dubbia l'ipotesi che il personaggio della variante stante sia interpretabile allo stesso modo: pur essendo le due iconografie simili, difatti, la differente postura potrebbe costituire una discriminante fondamentale, ad esempio fra l'ambito umano (personaggio stante: sacerdote?) e quello divino (seduto). Per il personaggio assiso su un trono sorretto da sfingi alate è stato proposto che possa trattarsi di Baal $\mathrm{Hammon}^{51}$ : in realtà, nel dossier l'unico reperto nel quale il tipo iconografico e il dio appaiono direttamente associati è la stele del tofet di Sousse; ulteriori indizi a favore di un'interpretazione di questo tipo provengono da due reperti rinvenuti nei tofet di Cartagine e Mozia, ma in questi casi a parte il trono con sfingi ai lati non è possibile ricostruire l'iconografia del personaggio ${ }^{52}$. A partire dal III-II sec. a.C. sono attestate in più occasioni iconografie che costituiscono un'elaborazione tarda del tipo analizzato finora e sembrano effettivamente rappresentare Baal $\mathrm{Hammon}^{53}$; ciò è ulteriormente testimoniato dal fatto che talvolta questi tipi iconografici vengono utilizzati per la rappresentazione di Saturno, che in età romana viene identificato con il dio feniciocartaginese $^{54}$. Va notato, tuttavia, che su un trono con sfingi alate possono essere assise

126, n. 57, tav. XXII); altri scarabei di provenienza incerta (fig. 13:24-26. Boardman 2003, 63, n. 17/26, tav. 17; 64, n. 17/39, tav. 17; 64, n. 17/40, tav. 17).

48 Gubel 1987, 126, n. 60, tav. XXII (scarabeo di diaspro verde scuro proveniente da una collezione privata); 136 , n. 82, tav. XXVII (scarabeo di corniola di provenienza cipriota). Cfr. anche 135, n. 77, tav. XXVI (scaraboide di agata di provenienza siriana, tra Homs e Palmira).

49 L'ipotesi del papiro è stata proposta per uno degli scarabei di Ibiza (fig. 13:9), quella della palma per un altro scarabeo ibicenco (fig. 13:8), quella del bocciolo di loto o della spiga di grano per la cosiddetta stele di Baal (fig. 13:3) e per l'anello d'oro di Utica (fig. 13:15).

50 Herrmann - Mallowan 1974, 92, n. 51, tav. LX (anche trono con sfingi alate alla base); 104-105, nn. 77-80, tav. XCII-XCIII (personaggio in trono con scettro "vegetale"); 105-106, nn. 84-85, tav. XCIV (pianta su alto stelo a sommità lanceolata simile alla "lancia" del personaggio della stele di Sulcis). Per l'interpretazione di tali scettri cfr. Gubel 1980.

51 Cfr. Bisi 1967, 94-95; Xella 1991, 112-130; D’Andrea 2014, 26.

52 Dal tofet di Cartagine proviene una statuetta di calcare databile alla prima metà del VI sec. a.C. che rappresenta un trono con sfingi ai lati su cui è assiso un personaggio di cui non si conserva praticamente nulla (cfr. Picard 1954, 37-38, Ca 8, tav. VIII; D'Andrea 2014, 51). Nelle trincee di spoliazione del sacello A del Tofet di Mozia è stato rinvenuto un tronetto frammentario di calcare caratterizzato da sfingi sui lati e datato fra la parte finale del VI e il V sec. a.C.: Ciasca 1996.

53 Ad esempio: una statuetta fittile proveniente dal santuario di Thinissut databile al II-I sec. a.C.; delle monete coniate alla fine del II sec. d.C. a Sousse/Hadrumetum con legenda Saeculum Frugiferum; due stele di II-I sec. a.C. provenienti da Costantina. Per un quadro complessivo dei reperti nei quali è attestato questo tipo iconografico: D'Andrea 2014, 315-316. Una statuetta di bronzo proveniente da Genoni, in Sardegna, e datata tra IV e III sec. a.C. raffigura un personaggio stante con copricapo turrito di piume, barba folta e braccio destro alzato con mano aperta in segno di saluto/benedizione (Xella 1991, 121, tav. 9:1); nella mano destra del personaggio era originariamente collocato un oggetto, verosimilmente un'asta/scettro.

54 Ad esempio in una coppa decorata proveniente da Chimtou (I-II sec. d.C.) e in una stele di Sidi Bou Rouis (II sec. d.C.): D’Andrea 2014, 316. 
diverse divinità, sia maschili che femminili ${ }^{55}$, e anche personaggi regali ${ }^{56}$; al tempo stesso, un personaggio molto simile a quello in esame può recare, sia nella variante stante $e^{57}$ che in quella assisa $^{58}$, un' ascia sulla spalla che viene tradizionalmente considerata un attributo di Melqart, si pensi in proposito alla stele di Bar-Hadad di Aleppo (metà IX sec. a.C.) ${ }^{59}$.

\section{MT.09.3004/S1}

L'ottavo reperto del lotto in esame è un piccolo frammento proveniente dalla pulizia dell'edificio b (US.3004; fig. 1:7). Il frammento, di arenaria calcarea, dovrebbe costituire l'angolo superiore destro o quello inferiore sinistro di un'edicola a doppio inquadramento semplice con betilo a rilievo (fig. 7:4-5; alt. 9,1 cm; largh. 6,7 cm; spess. $1 \mathrm{~cm}$ ); stele dello stesso tipo sono già attestate nel repertorio moziese (fig. 7:6 $)^{60}$.

\section{MT.09.M4/S1}

Il reperto era riutilizzato nell'angolo settentrionale di M.4 ed è stato lasciato in situ (fig. $1: 8 ; 11: 1-2)$. Si tratta di una lastra di calcare apparentemente integra con un apparato illustrativo non comune costituito da una sorta di betilo con due gambe (fig. 11:3; alt. 40 $\mathrm{cm}$; largh. $22 \mathrm{~cm}$; spess. 7,5 cm) ${ }^{61}$; un'iconografia simile compare su un'altra stele moziese attribuita allo strato III (fig. 11:4), dunque alla fase 6 (520 e 470 a.C.). In realtà, non si può escludere che il solco centrale sia una scheggiatura e che dunque sulla stele in esame fosse raffigurato un betilo di tipo tradizionale.

\section{CONCLUSIONI}

Le nove stele inedite esaminate in questo contributo, pur non essendo caratterizzate da elementi innovativi e peculiari rispetto al repertorio lapideo moziese già conosciuto, hanno permesso di fare il punto sulle caratteristiche di alcune tipologie formali e di alcune iconografie e sui confronti che esse trovano a Mozia e nei repertori lapidei degli altri tofet del Mediterraneo. La raffigurazione, su una delle stele del lotto, di un personaggio con copricapo conico, barba lunga, braccio destro alzato e mano aperta ha offerto l'opportunità di analizzare i caratteri, i confronti e le ipotesi interpretative proposte per questa iconografia, ampiamente attestata in ambito fenicio e punico sia nella resa stante che in quella assisa del personaggio.

55 Per qualche esempio in proposito si osservino gli scarabei riprodotti in Gubel 1987 e Boardman 2003.

56 Si pensi ad esempio al sarcofago di Ahiram di Biblo (Matthäus 2004, 330).

57 Cfr. ad esempio Gubel 1980, tav. II; 1987, 199-200, n. 153, tav. XL; Boardman 2003, 64, nn. 17/49, tav. 17; $66,17 / \mathrm{X} 19, \mathrm{pl} .54$.

58 Cfr. ad esempio Gubel 1980, tav. II; 1987, 46, n. 25, tav. VIII; D’Andrea 2014, 315-316, fig. 11.4, a.

59 Bonnet 1988, 132-136.

60 Moscati - Uberti 1981, 176-177, nn. 580-588, tav. LXXXVIII.

61 MT.09.M4/S1 (alt. $40 \mathrm{~cm}$; largh. $22 \mathrm{~cm}$; spess. 7,5 cm). 


\section{BIBLIOGRAFIA}

ACQUARO, E.

1969 Appunti su una stele da Sulcis: Oriens Antiqvvs 8 (1969), pp. 69-72.

BARTOLONI, P.

1976 Le stele arcaiche del tofet di Cartagine (Collezione di Studi Fenici 8), Roma 1976.

1986 Le stele di Sulcis. Catalogo (Collezione di Studi Fenici 24), Roma 1986.

BÉNICHOU-SAFAR, H.

2004a Le tophet de Salammbô à Carthage. Essai de reconstitution (Collection de l'École Française de Rome 342), Paris - Rome 2004.

2004b Le geste dit de l'orant sur les stèles puniques de Carthage: A. GonZaLEz PRATs (ed.), El mundo funerario. Actas del III Seminario Internacional sobre Temas Fenicios, Guardamar del Segura, mayo de 2002 (Homenaje al Prof. D. Manuel Pellicer Catalán), Alicante 2004, pp. 99-116.

Bevilacqua, F. - Ciasca, A. - Matthiae Scandone, G. - Moscati, S. - Tusa, V. - Tusa Cutroni, A.

1972 Mozia - VII. Rapporto preliminare della Missione congiunta con la Soprintendenza alle Antichità della Sicilia Occidentale (Studi Semitici 40), Roma 1972.

BISI, A.M.

$1967 \quad$ Le stele puniche (Studi semitici 27), Roma 1967.

BOARDMAN, J.B.

2003 Classical Phoenician Scarabs. A catalogue and study (British Archaeological Reports International Series 1190, Studies in Gems and Jewell II), Oxford 2003.

BONDì, S.F.

$1972 \quad$ Le stele di Monte Sirai (Studi Semitici 43), Roma 1972.

BONNET, C.

1988 Melqart. Cultes et mythes de l'Héraclès tyrien en Méditerranée (Studia Phoenicia VIII), Leuven 1988.

Brancoli, I. - Ciasca, A. - Garbini, G. - Pugliese, B. - Tusa Cutroni, A. - Tusa, V.

1967 Mozia - III. Rapporto preliminare della Missione archeologica della Soprintendenza alle Antichità della Sicilia Occidentale e dell'Università di Roma (Studi Semitici 24), Roma 1967.

CiAsca, A.

1992 Mozia: sguardo d'insieme sul tofet: Vicino Oriente 8 (1992), pp. 113-155.

1996 Un arredo cultuale del tofet di Mozia (Sicilia): E. ACQUARO (ed.), Alle soglie della classicità: il Mediterraneo tra tradizione e innovazione. Studi in onore di Sabatino Moscati, 2, Pisa - Roma 1996, pp. 629-637.

Ciasca A. - Forte, M. - Garbini, G. - Moscati, S. - Pugliese, B. - Tusa, T.

1964 Mozia - I. Rapporto preliminare della Missione archeologica della Soprintendenza alle Antichità della Sicilia Occidentale e dell'Università di Roma (Studi Semitici 12), Roma 1964.

Ciasca, A. - Guzzo Amadasi, M.G. - Moscati, S. - Tusa, V.

1970 Mozia - VI. Rapporto preliminare della Missione congiunta con la Soprintendenza alle Antichità della Sicilia Occidentale (Studi Semitici 37), Roma 1970.

Ciasca, A. - Tusa, V. - Uberti, M.L.

1973 Mozia - VIII. Rapporto preliminare della Missione congiunta con la Soprintendenza alle Antichità della Sicilia Occidentale (Studi Semitici 45), Roma 1973.

CuLican, W.

1970 Problems of Phoenicio-Punic Iconography. A Contribution: The Australian Journal of Biblical Archaeology 1/3 (1970), pp. 28-57. 
D'ANDREA, B.

2014 I tofet del Nord Africa dall'età arcaica all'età romana (VIII sec. a.C. - II sec. d.C.). Studi archeologici (Collezione di Studi Fenici 45), Roma 2014.

D'ANDREA, B. - GIARDINO, S

2013 Il tofet dove e perché. L'identità fenicia, il Circolo di Cartagine e la fase Tardo Punica: Bollettino di Archeologia on-line 4 (2013), pp. 1-29.

GuBEL, E.

1980 An Essay on the Axe-Bearing Astarte and Her Role in a Phoenician «Triad»: Rivista di Studi Fenici 8 (1980), pp. 1-17.

1987 Phoenician Furniture. A Typology based on Iron Age Representations with Reference to the Iconographical Context (Studia Phoenicia VII), Leuven 1987.

HerRmann, G. - MALlOWAN, M.

$1974 \quad$ Ivories from Room SW 37. Fort Salmaneser. Commentary, Catalogue and Plates (Ivories MATTHÄUS, $\mathrm{H}$ from Nimrud, 1949-1963, fasc. III), London 1974.

2004 Kunst und Kunsthandwerk: S. PETER (ed.), Hannibal ad portas: Macht und Reichtum Karthagos. Begleitbuch zur Sonderausstellung im Badischen Landesmuseum Karlsruhe, Karlsruhe - Stuttgart 2004, pp. 330-335.

MOSCATI, S.

1967 Iconografie fenicie a Mozia: Rivista degli Studi Orientali 42 (1967), pp. 61-64.

$1990 \quad$ L'arte dei Fenici, Milano 1990.

MOSCATI, S. - UBERTI, M.L.

1970 Le stele puniche di Nora nel Museo Nazionale di Cagliari (Studi Semitici 35), Roma 1970.

1981 Scavi a Mozia - Le stele (Pubblicazioni del Centro di Studio per la Civiltà fenicia e punica 23; Serie Archeologica 25), 1-2, Roma 1981.

1985 Scavi al tofet di Tharros, i monumenti lapidei (Collezione di Studi Fenici 21), Roma 1985.

NigRO, L.

2004 Il tofet: L. Nigro - G. Rossoni (edd.), "La Sapienza” a Mozia. Quarant'anni di ricerca archeologica, 1964-2004. Catalogo della Mostra, Università di Roma "La Sapienza”, Facoltà di Scienze Umanistiche, Museo dell'Arte Classica, 27 febbraio-18 maggio 2004, Roma 2004, pp. 38-45.

2012 Scavi e restauri dell'Università di Roma "La Sapienza" a Mozia, 2007-2009: il Tempio del Kothon, il Temenos Circolare, il Sacello di Astarte e il Tofet: C. AMPOLO (ed.), Sicilia occidentale. Studi, rassegne, ricerche. Atti delle settime giornate di studi sull'area elima e la Sicilia occidentale nel contesto mediterraneo, Erice 12-15 ottobre 2009 (Seminari e convegni 29), Pisa 2012, pp. 207-218.

2013 Mozia: il Tofet e la città. Il limite meridionale del santuario e le strutture collegate negli scavi della Sapienza 2010-2011: Scienze dell’Antichità 19/1 (2013), pp. 37-53.

PETERS, S. (ed.)

2004 Hannibal ad portas: Macht und Reichtum Karthagos. Begleitbuch zur Sonderausstellung im Badischen Landesmuseum Karlsruhe, Karlsruhe - Stuttgart 2004.

PICARD, C.

$1954 \quad$ Catalogue du Musée Alaoui. Nouvelles séries, 1-2, Paris 1954.

WILSON, V.

1974 Excavations at Kouklia (Paleopaphos). The Kouklia Sanctuary, Appendix: Report of Department of Antiquities in Cyprus 1974 (1974), pp. 139-146. 
Xella, P.

1991 Baal Hammon. Recherches sur l'identité et l'histoire d'un dieu phénico-punique (Collezione di Studi Fenici 32; Contributi alla Storia della Religione Fenicio-Punica I), Roma 1991. 

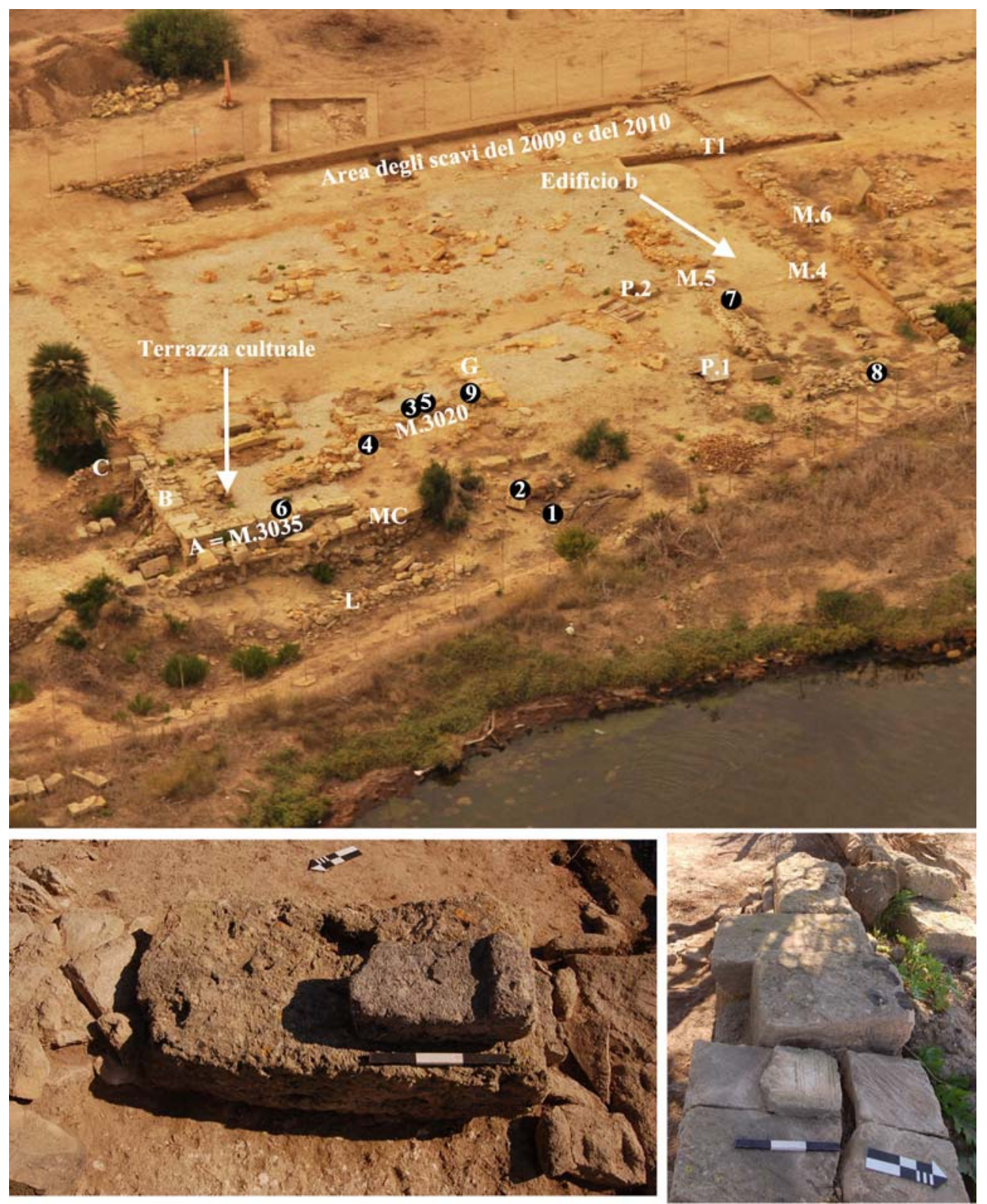

Fig. 1 - In alto: Mozia, parte centrale del Tofet, foto aerea da nord. In basso a sinistra: particolare di M.3020, foto da nord-ovest, in primo piano la stele MT.09.M3020/S4 (n. 3), appena ad ovest MT.09.3020/S1 (n. 5). In basso a destra: particolare di M.3035, foto da est, in primo piano MT.09.M3035/S1 (n. 6). La foto in alto è di L. Nigro (rielaborata dall'autore), quelle in basso di N. Chiarenza. 
XVIII (2014)
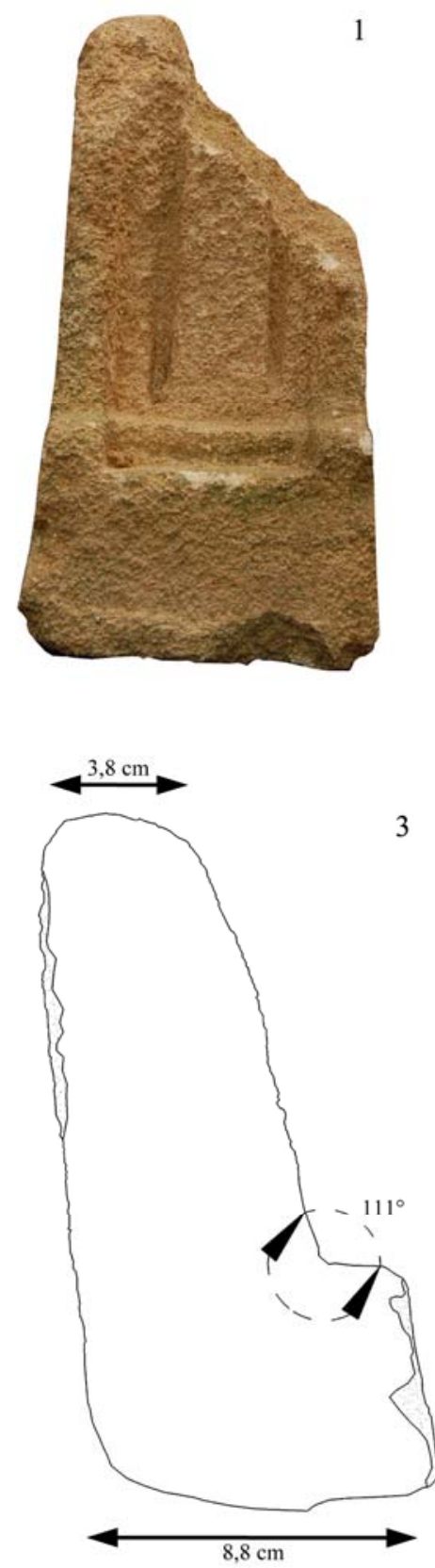

Nuove stele dal Tofet di Mozia
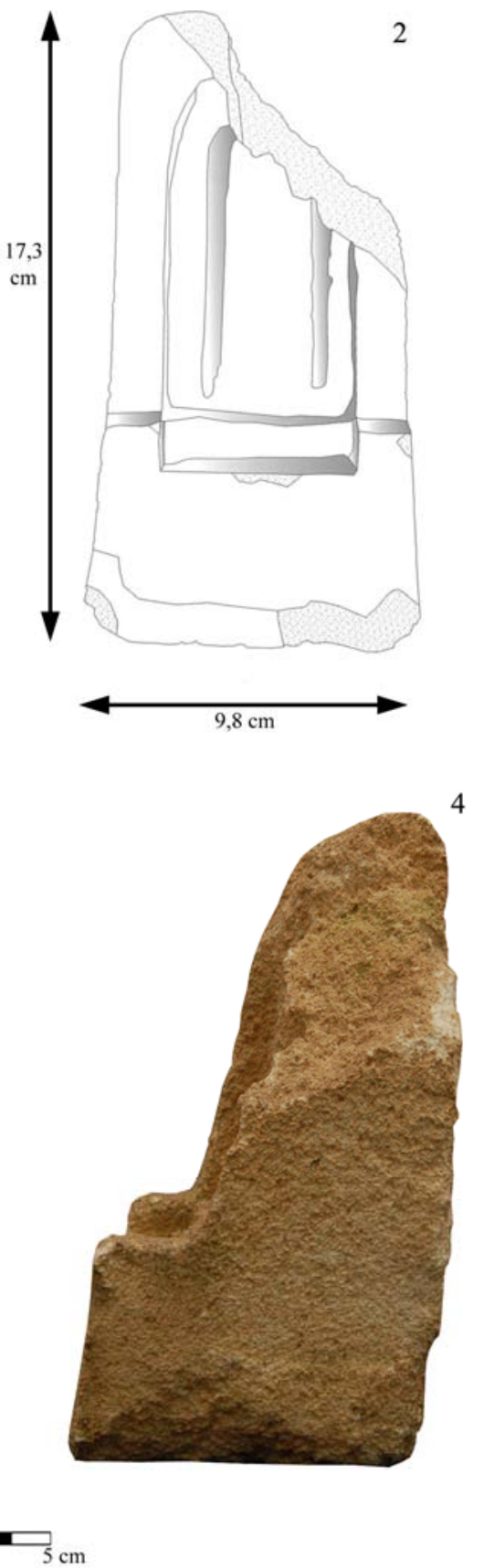

Fig. 2 - Mozia, Tofet: foto e disegni della stele MT.09.3011/S1 (foto e disegni dell'autore). 

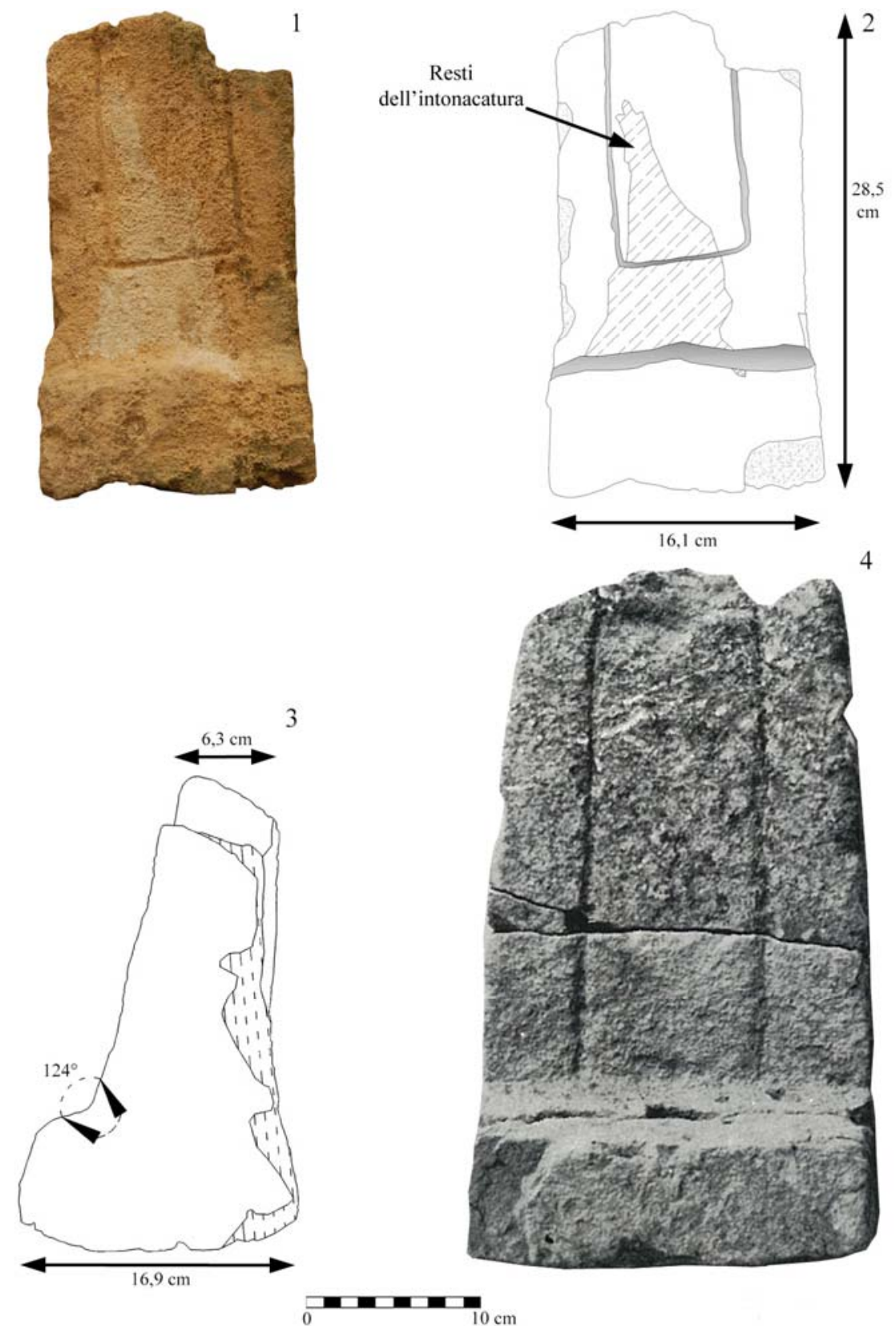

Fig. 3 - Mozia, Tofet: foto e disegni della stele MT.09.3011/S3 (nn. 1-3: foto e disegni dell'autore). Confronto dal repertorio lapideo moziese (n. 4: Moscati - Uberti 1981, 112, n. 188, tav. XXIV). 

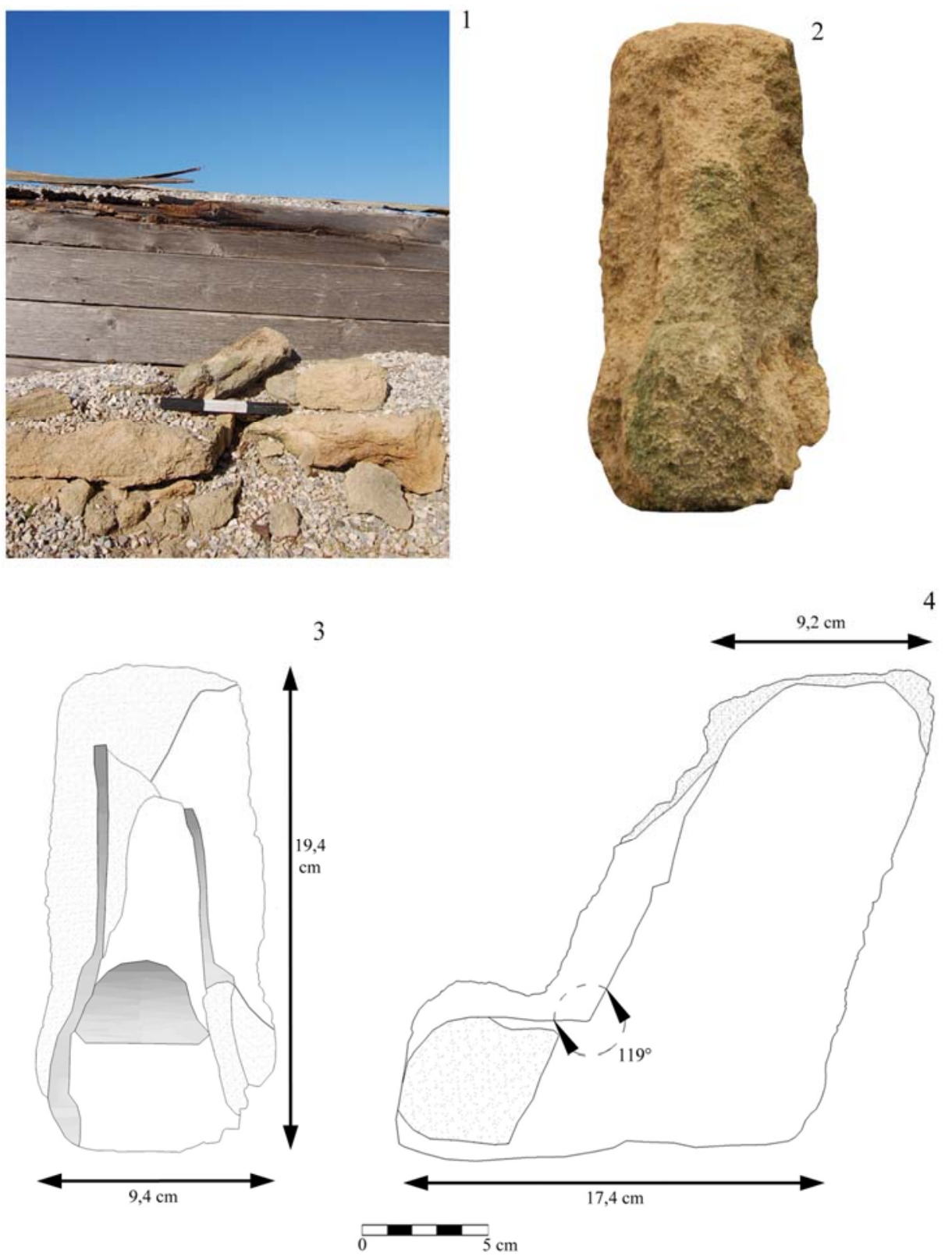

Fig. 4 - Mozia, Tofet: foto del muro G, da est; in primo piano la stele MT.09.3011/S2, sullo sfondo una delle costruzioni lignee installate per la conservazione del santuario e rimosse nel 2009 (n. 1: foto di N. Chiarenza). Foto e disegno della stele MT.09.3011/S2 (nn. 2-4: foto e disegni dell'autore). 

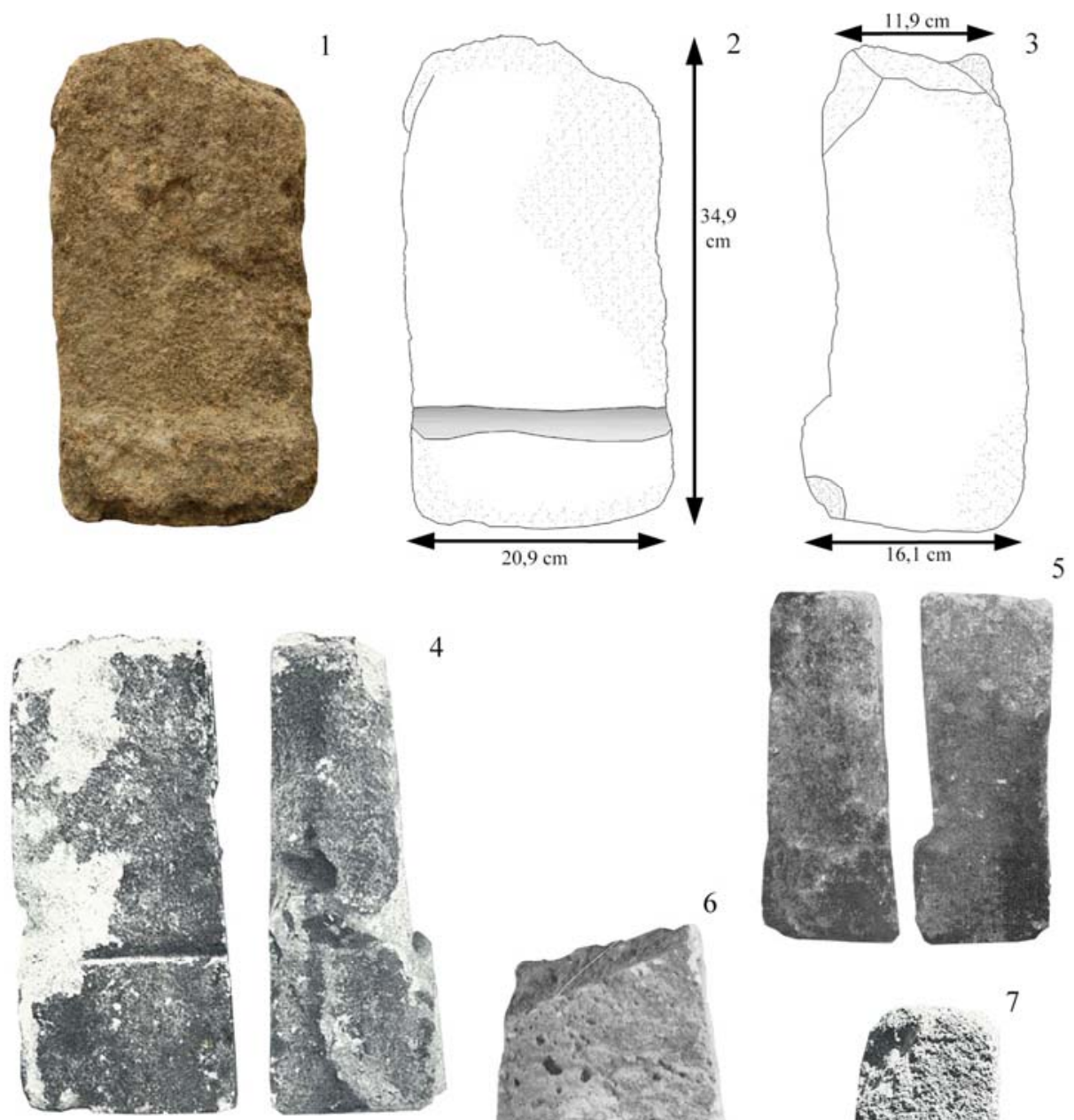

4
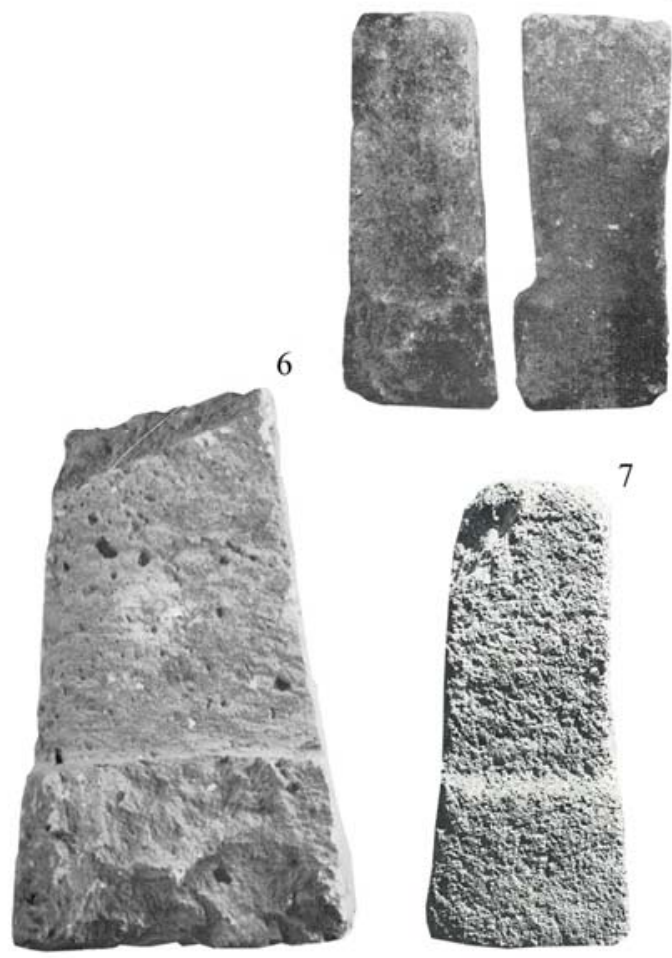

Fig. 5 - Mozia, Tofet: foto e disegni della stele MT.09.M3020/S4 (nn. 1-3: foto e disegni dell'autore). Cippi a trono vuoti, con profilo a L, dai Tofet di Mozia (nn. 4-5: Moscati Uberti 1981, 95, n. 68, tav. IX; 96, n. 78, tav. XI), Sulcis (n. 6: Bartoloni 1986, 36, n. 49, tav. VII) e Cartagine (n. 7: Bartoloni 1976, 86, n. 54, tav. XV). 

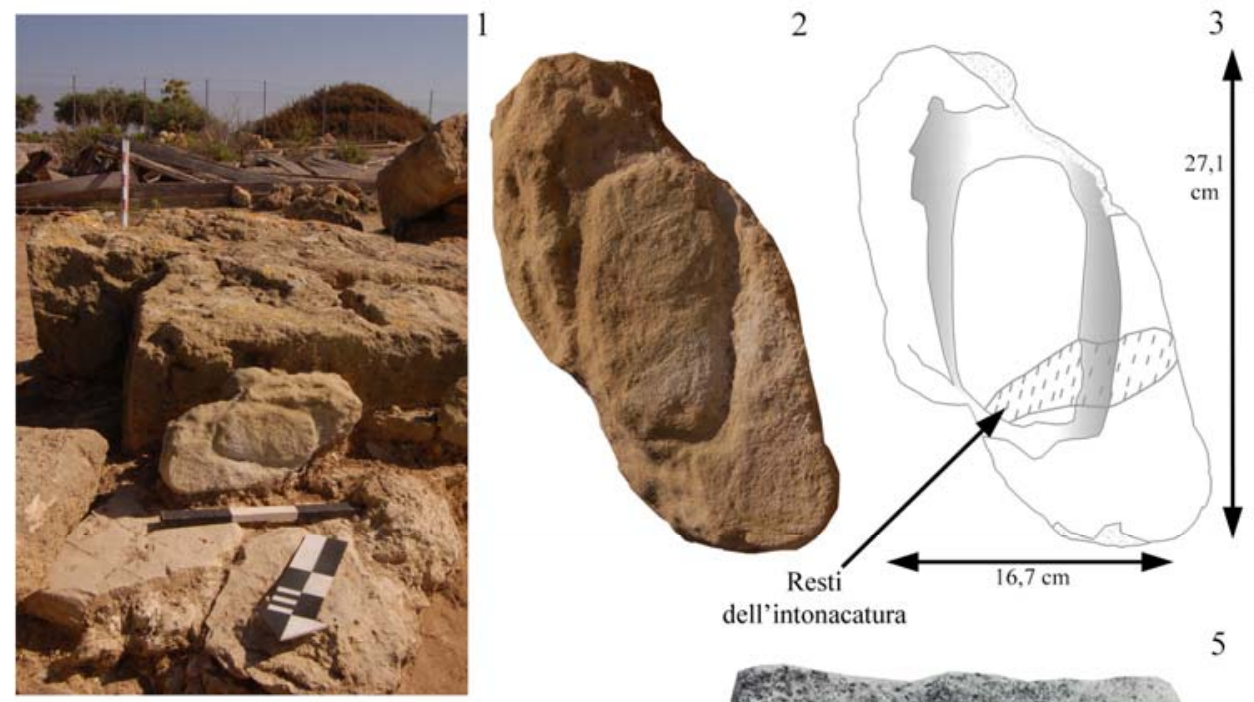

4
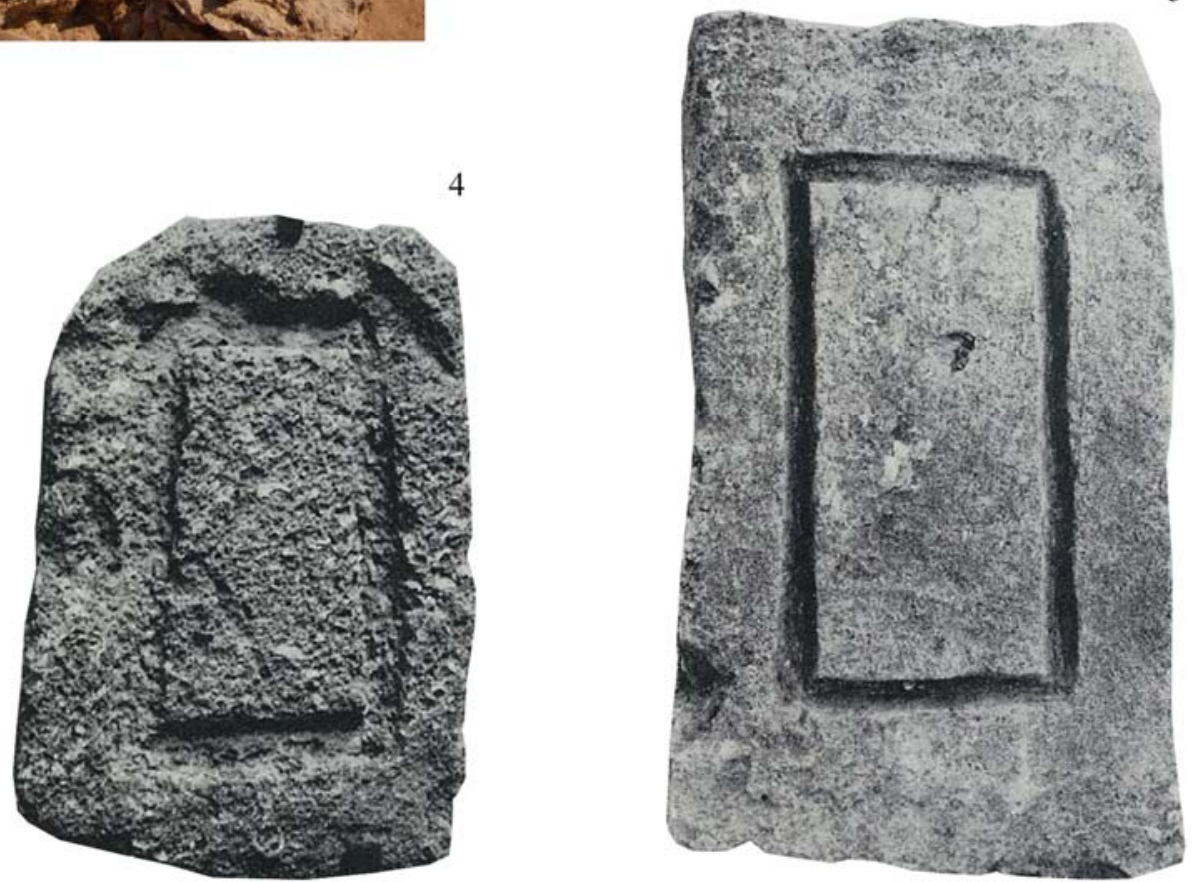

대드.

Fig. 6 - Mozia, Tofet: foto di MT.09.M3020/S3, da nord (n. 1: foto di N. Chiarenza); foto e disegno della stele (nn. 2-3: foto e disegno dell'autore). Altri esempi della variante a del tipo dal Tofet di Mozia (nn. 4-5: Moscati - Uberti 1981, 144, n. 383, tav. LVII; 139, n. 352, tav. LIII). 


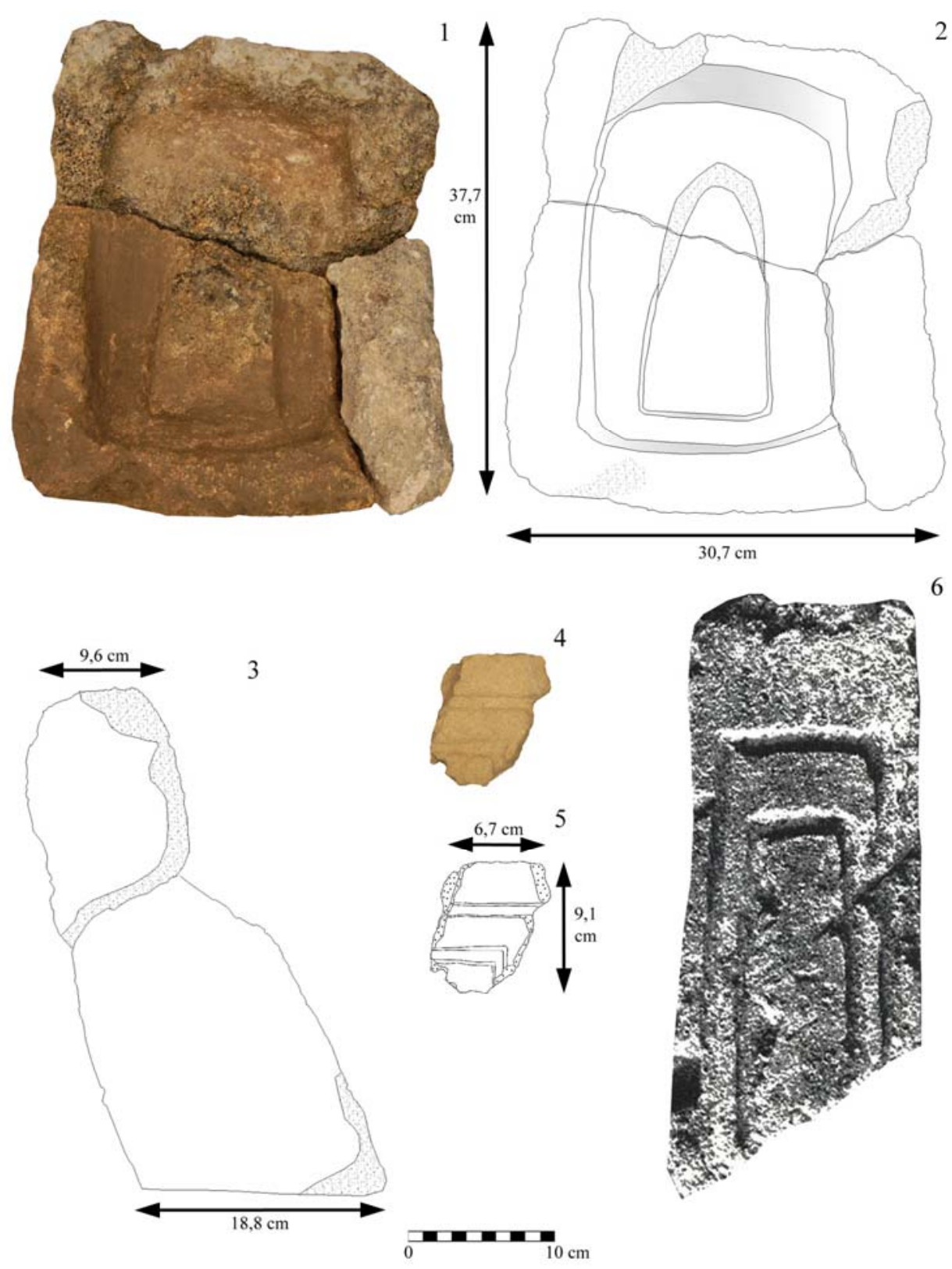

Fig. 7 - Mozia, Tofet: foto e disegni della stele MT.09.M3020/S1 (nn. 1-3: foto e disegni dell'autore); foto e disegno di MT.09.3004/S1 (nn. 4-5: foto e disegno dell'autore). Confronto dal repertorio lapideo moziese (n. 6: Moscati - Uberti 1981, 176, n. 583, tav. LXXXVIII). 


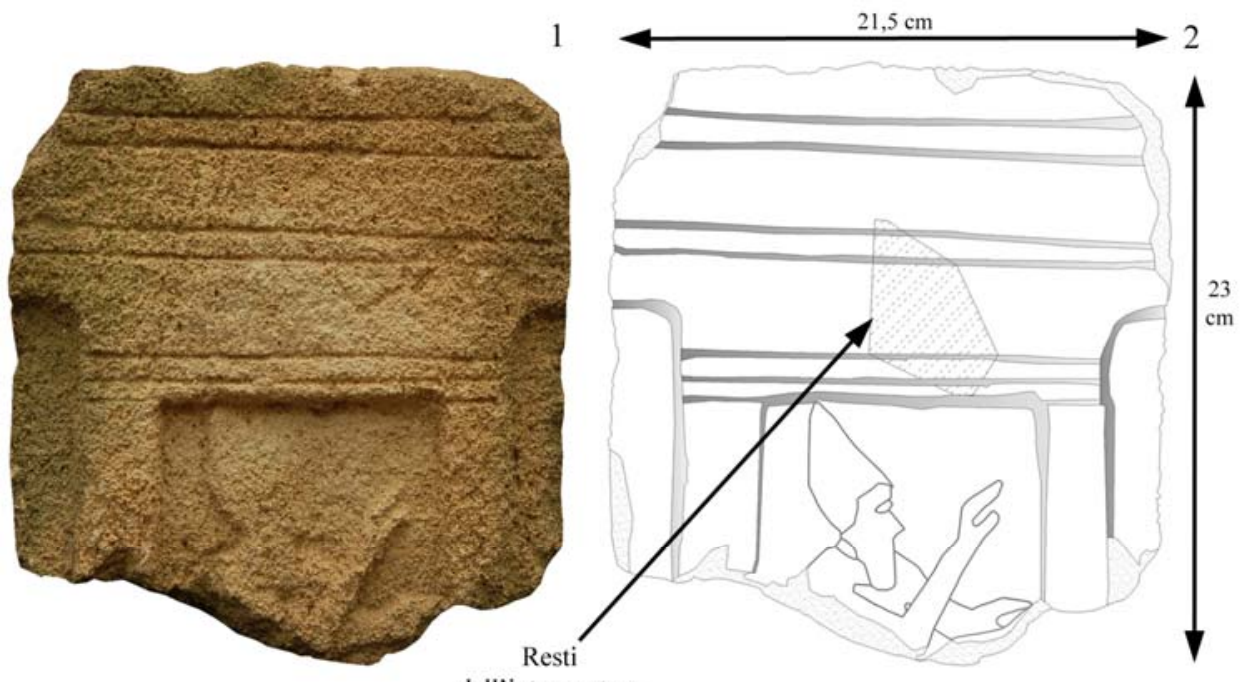

dell'intonacatura

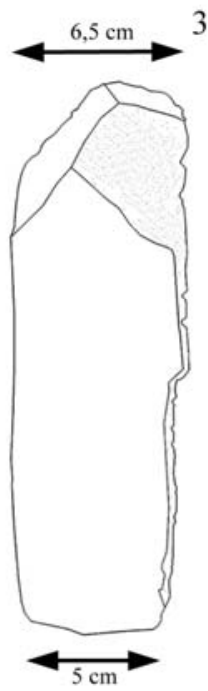

4

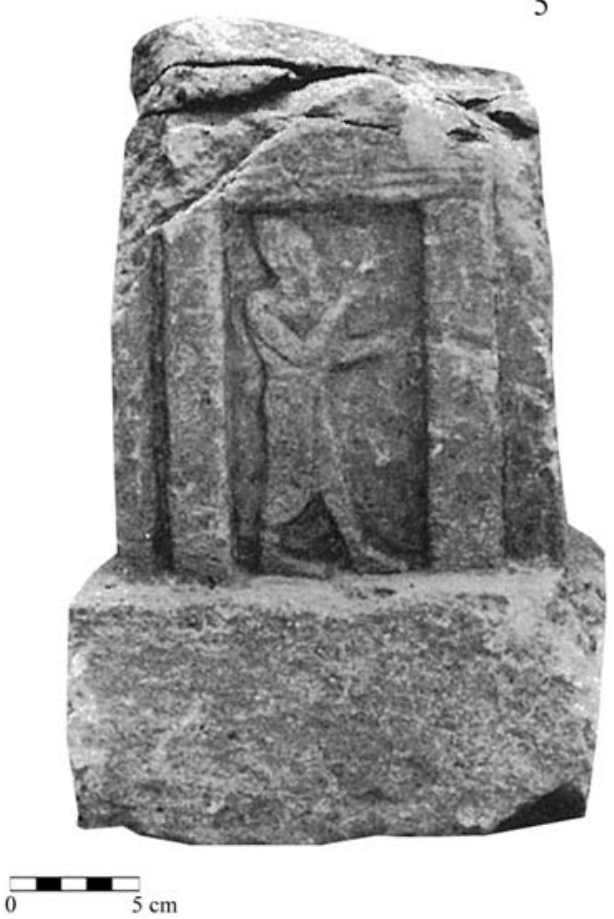

Fig. 8 - Mozia, Tofet: foto e disegni della stele MT.09.M3035/S1 (nn. 1-4: foto e disegni dell'autore). Confronto dal repertorio lapideo moziese (n. 5: Moscati - Uberti 1981, 243, n. 924, tav. CLXV). 


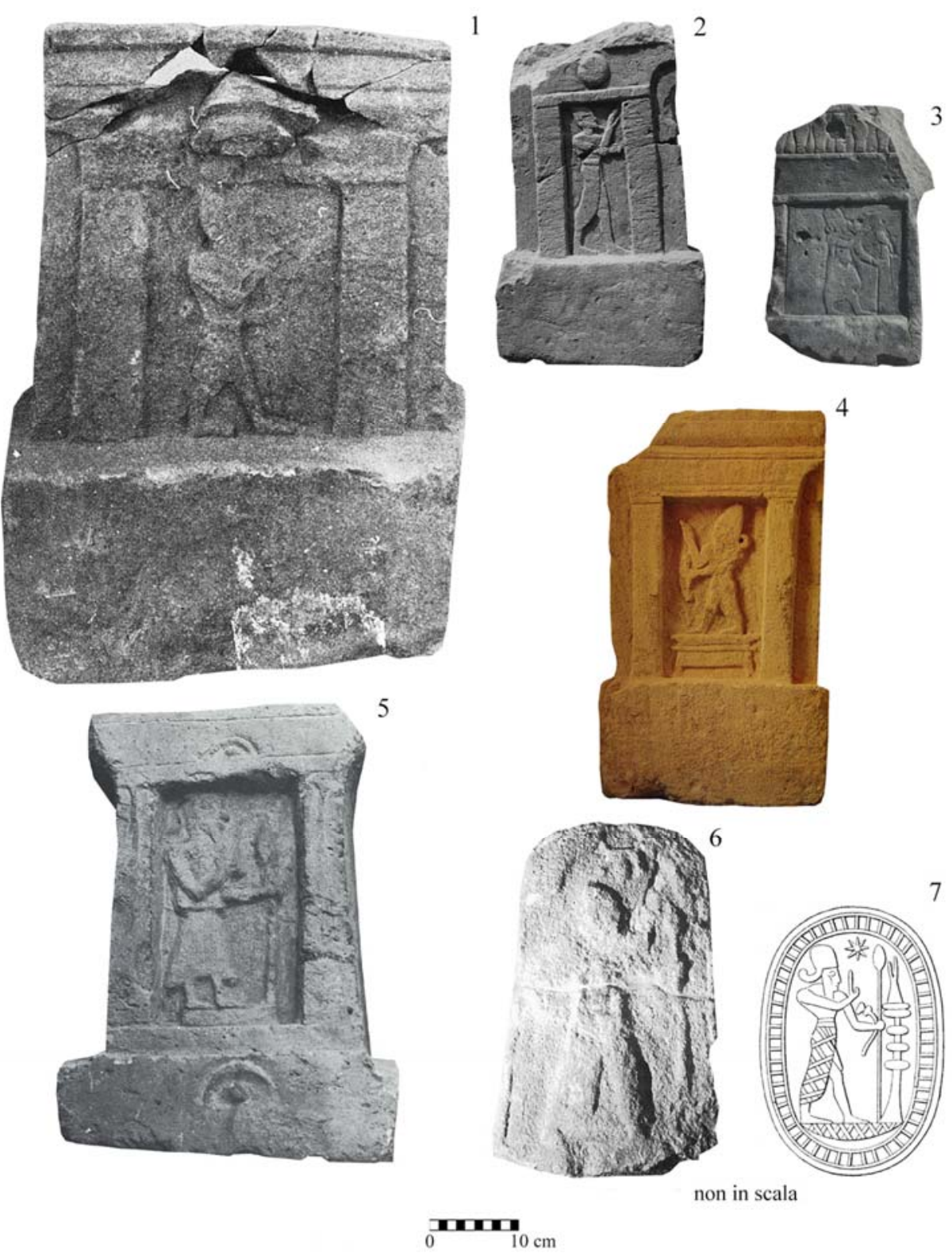

Fig. 9 - Confronti per il personaggio rappresentato su MT.09.M3035/S1: stele dal Tofet di Mozia (nn. 1-4: Moscati - Uberti 1981, 243, n. 925, tav. CLXV; Moscati 1990, fig. 69; Moscati - Uberti 1981, 248, n. 947, tav. CLXX; Moscati 1990, fig. 68), dal tofet di Sulcis (n. 5: Bartoloni 1986, 56, n. 194, tav. XXXII) e da Kouklia (n. 6: Wilson 1974, tav. XXI, 6); scarabeo di probabile provenienza cipriota (n. 7: Acquaro 1969, 70-71, fig. 1). 

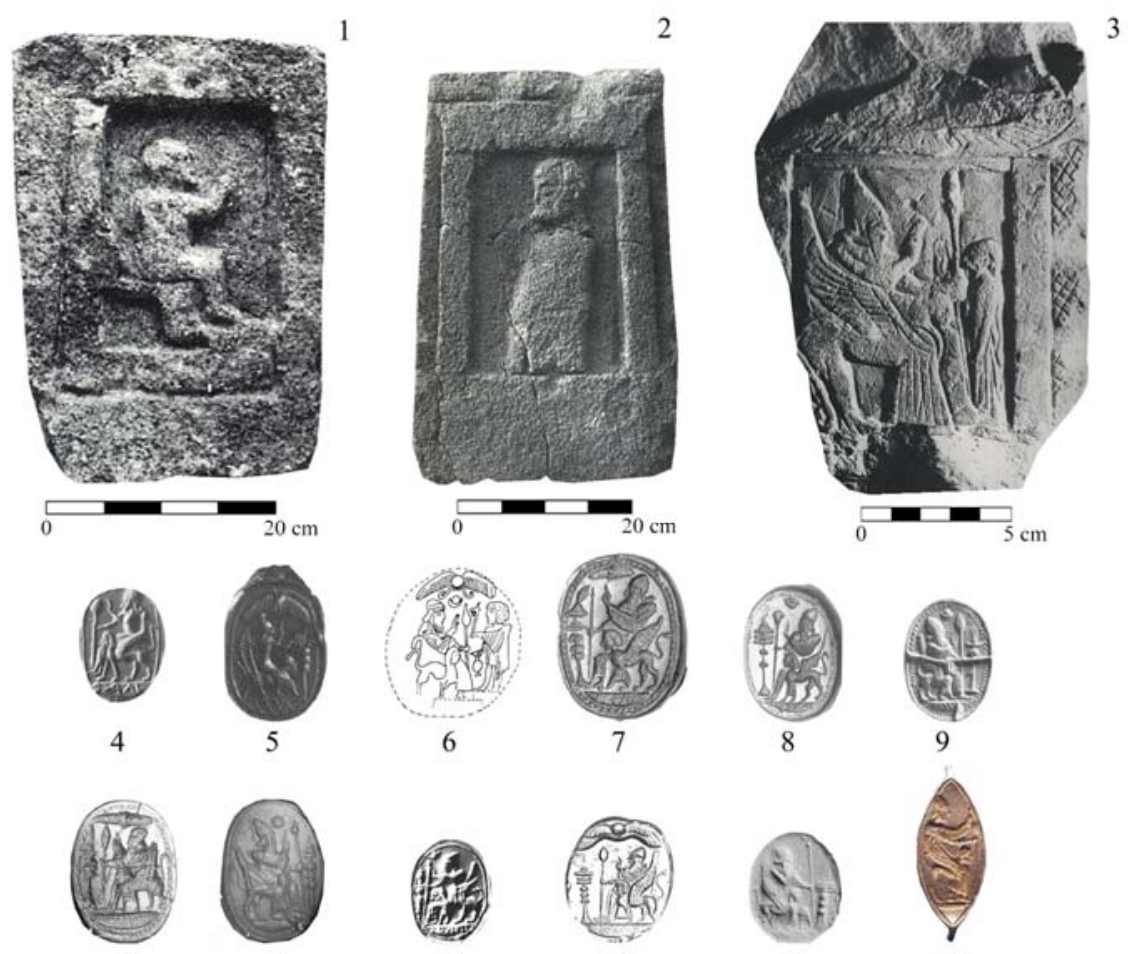

12

13
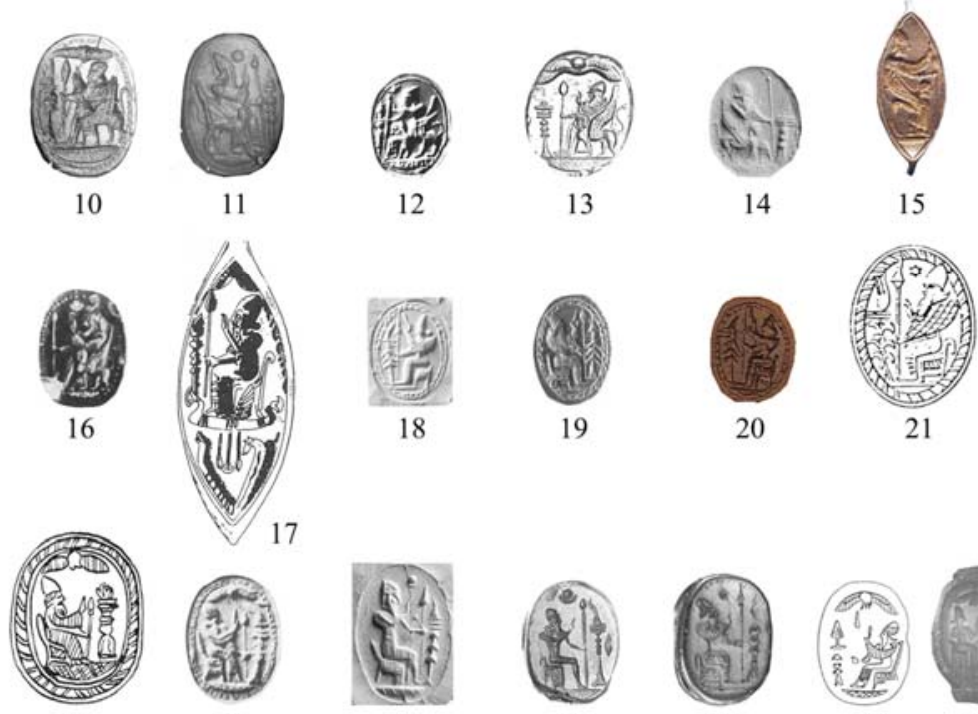

23
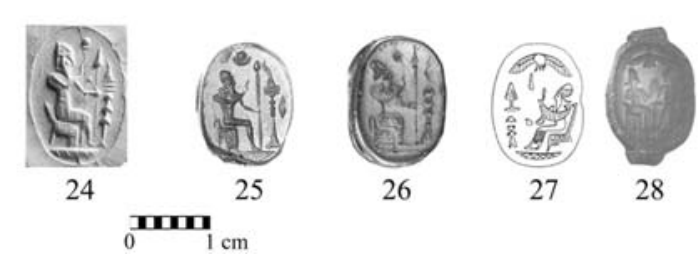

Fig. 10 - Personaggio con barba e copricapo seduto su un trono: stele dai Tofet di Mozia (nn. 1-2: Moscati - Uberti 1981, 259, n. 1005, tav. CLXXXIV; 259-260, n. 1007, tav. CLXXXV) e Sousse (n. 3: Picard 1954, Cb 1075, tav. CXXVI); scarabei e gioielli di varia provenienza (nn. 4-28: per la bibliografia dei singoli reperti si vedano le note 41 e 43-52). 


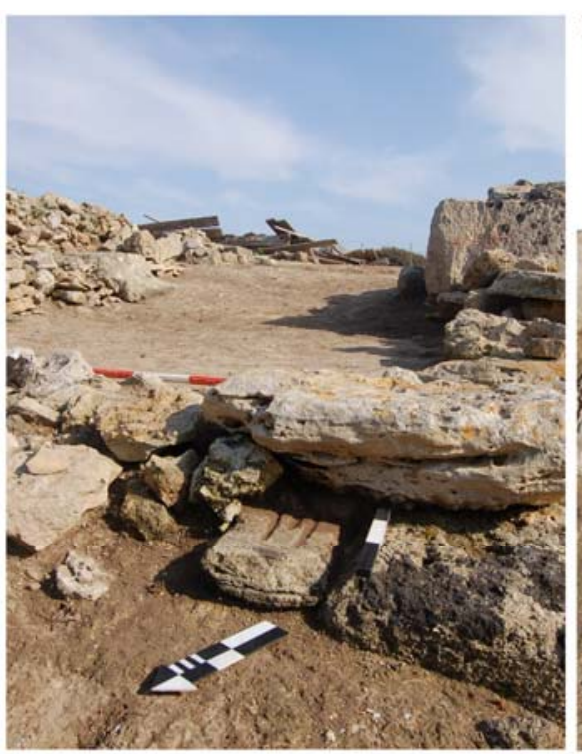

1
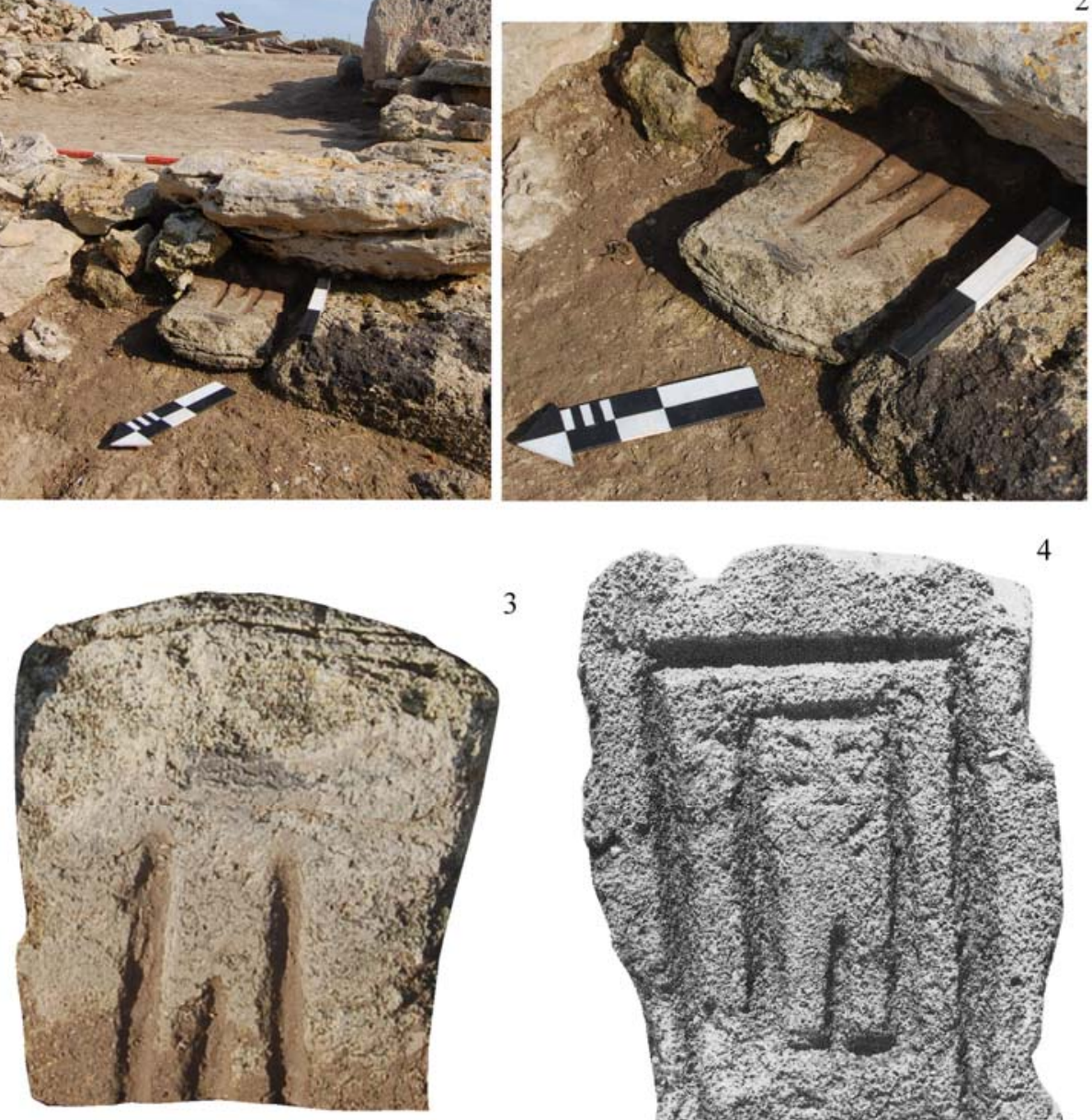

3

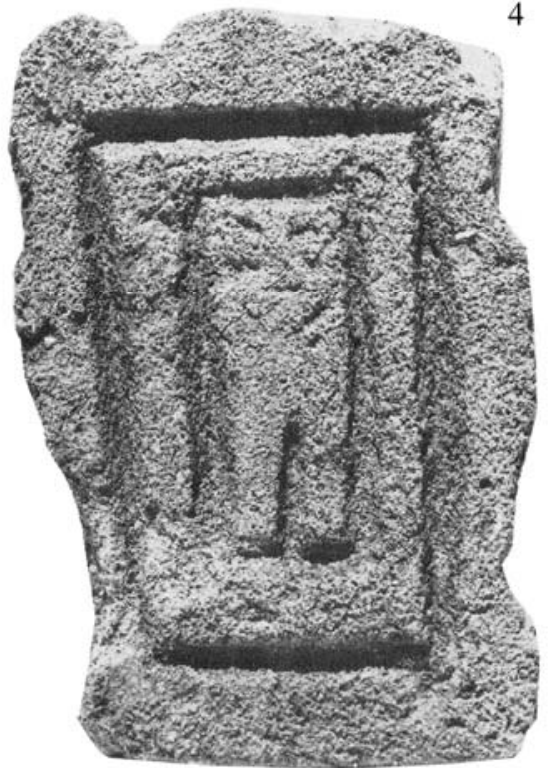

0

$10 \mathrm{~cm}$

Fig. 11 - Mozia, Tofet: foto della stele MT.09.M4/S1 riutilizzata nell'angolo settentrionale di M.4, da nord-ovest (nn. 1-2: foto di N. Chiarenza); foto della faccia anteriore (n. 3: foto rielaborata dall'autore). Confronto dal repertorio lapideo moziese (n. 4: Moscati - Uberti 1981, 254, n. 978, tav. CLXXIX). 